\title{
Case reports of antegrade and retrograde iatrogenic coronary spiral dissection complicating percutaneous coronary intervention: Keep calm and carry on!
}

\author{
Riccardo Iorio $^{1}$, Giuseppe Terlizzese ${ }^{1}$, Alessandra Pizzuto ${ }^{1}$ Ernesto Greco $^{2}$ and Giuseppe Massimo Sangiorgi $^{3 *}$ \\ ${ }^{1}$ Department of Cardiology, Cardiac Cath Lab, Clinica San Gaudenzio, Novara Italy \\ ${ }^{2}$ Department of Cardiothoracic Surgery, University La Sapienza, Rome, Italy \\ ${ }^{3}$ Department of Biomedicine and Prevention, Division of Cardiology, University of Tor Vergata, Italy
}

\begin{abstract}
Iatrogenic dissection during PCI represents one of the most fearing complication of interventional cardiology, since abrupt hemodynamic instability and patient compromise may occur related to coronary occlusion. In this emergency situation, different hemodynamic support devices and interventional skills, are needed in order to promptly recover the patient from cardiogenic shock, especially when the left coronary three is involved by the dissection. We report herein two different iatrogenic retrograde, and antegrade dissections, one involving both left anterior descending artery, left circumflex and aortic bulb and the second characterized by abrupt closure of left main associated with severe hemodynamic compromise and ventricular fibrillation storm which required continuous DC shocks, Impella and ECMO devices support to stabilize the patient. Both cases were successfully managed percutaneously. Different techniques and tricks in order to re-open the vessels are described. We conclude that in case of iatrogenic coronary dissection, a "keep calm and carry on" strategy should be adopted by the interventional cardiologist team in order to solve such dramatic complication.
\end{abstract}

\section{Introduction}

Iatrogenic coronary spiral dissection is a potential complication during coronary angiography or percutaneous coronary intervention (PCI) that can lead to emergency surgical aortic repair, coronary artery bypass graft surgery $(\mathrm{CABG})$ or death. Although rare, with a reported incidence range between $0.02 \%$ and $0.08 \%$ in diagnostic procedures and between $0.07 \%$ and $0.6 \%$ during percutaneous coronary interventions $[1,2]$ its occurrence can have devastating consequences if not promptly treated. In case of retrograde dissection involving LM, abrupt vessel closure and cessation of blood flow towards a large portion of myocardium resulting in haemodynamic collapse, often occurs. In this setting, re-opening of the true lumen is extremely difficult and technical expertise is needed to find a tailored management strategy. We report herein two cases underlying technical solutions to rewire the true lumen and re-establish vessel patency.

\section{Case report 1}

A 77-year-old Caucasian woman affected by long-term systolic hypertension experienced typical angina and syncope during exercise and was admitted to the ED. Troponin-I was $41.9 \mathrm{ng} / \mathrm{L}$ (n.v. $<20 \mathrm{ng} / \mathrm{L}$ ). Coronary angiography showed an hypoplastic right coronary artery (RCA- Figure1 - Panel A), dominant left circumflex coronary artery (LCX - Figure 1 - Panel B) without significant stenosis and left anterior descending (LAD) artery with a long sub-occlusion from the middle segment to the apex. First diagonal branch was normal and a large septal branch with angulated take-off was involved in the diseased segment (Figure 1- Panel C). Percutaneous angioplasty of LAD was performed utilizing a 3.5 Extra Backup 6F guiding catheter (Medtronic, Santa Rosa, CA, USA), and a Sion Blue 0.014" guidewire (Asahi Intecc Co.,
Aichi, Japan) was placed distally in the LAD. A Balance Middleweight guidewire (Abbott Diagnostics, Lake Forest, Illinois) was advanced into septal branch utilizing a Super Cross $90^{\circ}$ microcatheter (Teleflex Medical OEM, Gurnee, Illinois) due to the acute take-off of the vessel (Figure 2 white arrow- Panel A). Then, a balloon (Euphora $2.5 \times 20 \mathrm{~mm}$; Medtronic, Santa Rosa, CA, USA) was inflated to nominal pressure (8 atm) to predilate LAD segment. At control angiography, iatrogenic spiral dissection of LAD was evident with retrograde extension into LM and origin of the aorta (Dunning Classification Type II [3]; Figure 2 - Panel B). Since patient's hemodynamic was stable, a DES Ultimaster Tansei $2.75 \times 13 \mathrm{~mm}$ (Terumo corporation, Tokyo, Japan) followed by Ultimaster Tansei 3.0 x $28 \mathrm{~mm}$ were deployed in an overlapping fashion (Figure 2 - Panel C) in the mid and proximal LAD segment. Then, PCI of LM was performed by implanting a first Ultimaster Tansei 3.5 x $9 \mathrm{~mm}$ stent at 12 atm followed by a second overlapped Ultimaster Tansei $3.5 \times 12 \mathrm{~mm}$ stent at $18 \mathrm{~atm}$ up to aortic root origin, since after the first LM stent, a free intimal flap with staining contrast was still present (Figure 2 - Panel D). Then, extension of the spiral dissection to the origin of LCX, probably due to flap extension after LM stenting at high pressure inflation (Figure 2 - Panel E), was noticed. Thus, a

${ }^{\star}$ Correspondence to: Giuseppe Massimo Sangiorgi, Department of Biomedicine and Prevention, Division of Cardiology, University of Tor Vergata, Rome, Italy, E-mail: gsangiorgi@gmail.com

Key words: iatrogenic coronary dissection, cardiogenic shock, balloon litotripsy, case report

Received: March 02, 2021; Accepted: March 15, 2021; Published: March 25, 2021 
Choice PT Extra Support 0.014' guidewire was utilized in order to gain the true distal lumen of the LCX with a minimal subintimal and reentry ("mini-STAR") technique (4), and the procedure completed with a DES Ultimaster Tansei $3.5 \times 18 \mathrm{~mm}$ stent at the ostium of the LCX with $\mathrm{T}$ stent technique at 18 atm with optimal immediate result (Figure 2 - Panel F). The patient was submitted to CCU and five days later an angio-CT scan demonstrated complete sealing of aortic dissection with complete stent patency (Figure 2 - Panel G). Patient was discharged after ten days. A control angiography performed 6 months later after readmission for typical chest pain and positive sub-maximal exercise stress test demonstrated complete stents patency and TIMI 3 flow of both LAD and LCX (Figure 3 - Panel A, B and C).
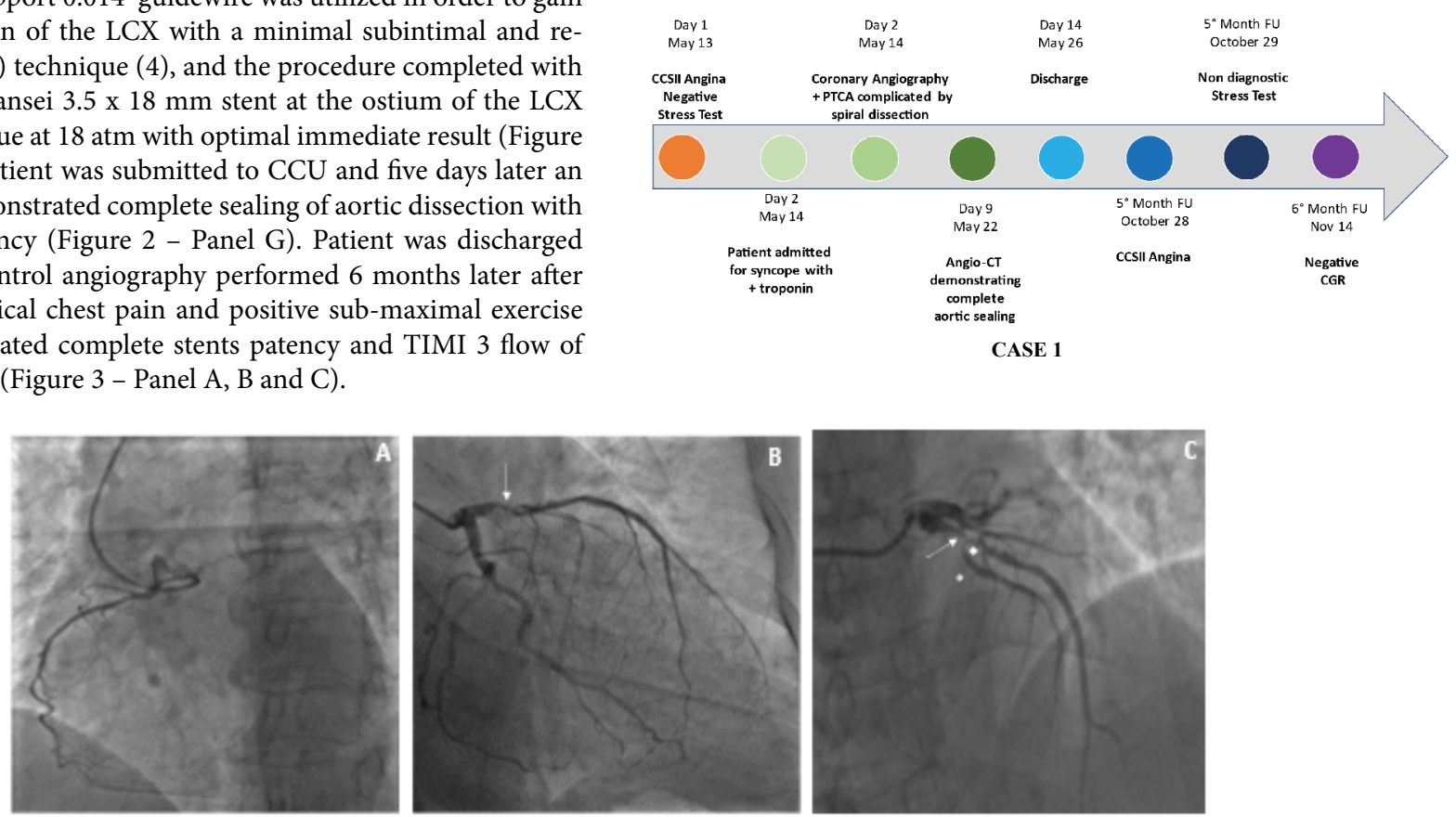

Figure 1. Basal coronary angiography of Case-1, showing hypoplastic RCA (Panel A), dominant LCX without significant stenosis (Panel B) and LAD with long sub-occlusion and bifurcating with a first diagonal branch and a septal branch compatible with "dual" LAD (Panel C).
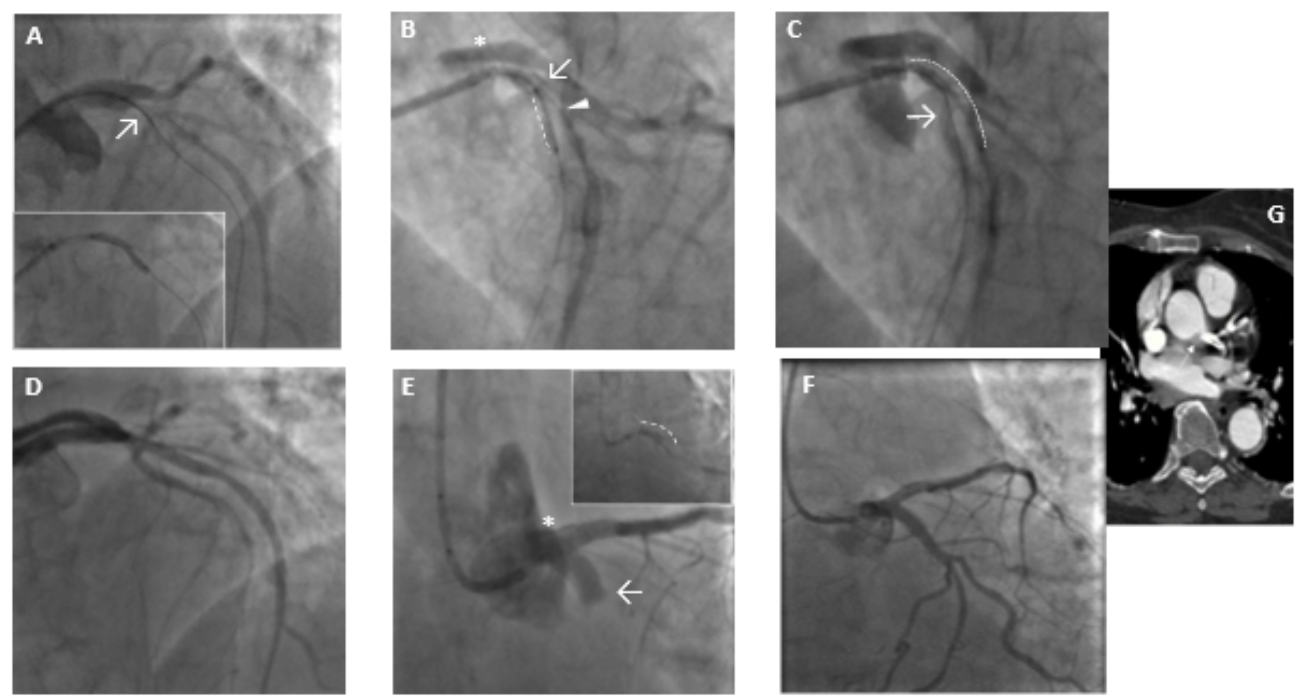

Figure 2. Case-1: PTCA balloon dilation in LAD stenotic segment and guidewire to protect the septal large branch by the mean of a microcatheter ( $\rightarrow$ ) (Panel A). Retrograde spiral dissection of the LAD $(*)$ (Dunning Classification Type II; Panel B). Stenting of septal branch and proximal LAD segment (...) (Panel B/C). Stenting of left main up to aortic root origin (Panel D). Extension spiral dissection and occlusion of LCX from the ostium $(\rightarrow)$ (Panel E). Successful and complete closure of the dissection tear associated to TIMI 3 flow (Panel F). Angio-CT scan demonstrated complete sealing of aortic dissection with complete stent patency $(\square)$ (Panel G) before discharge.
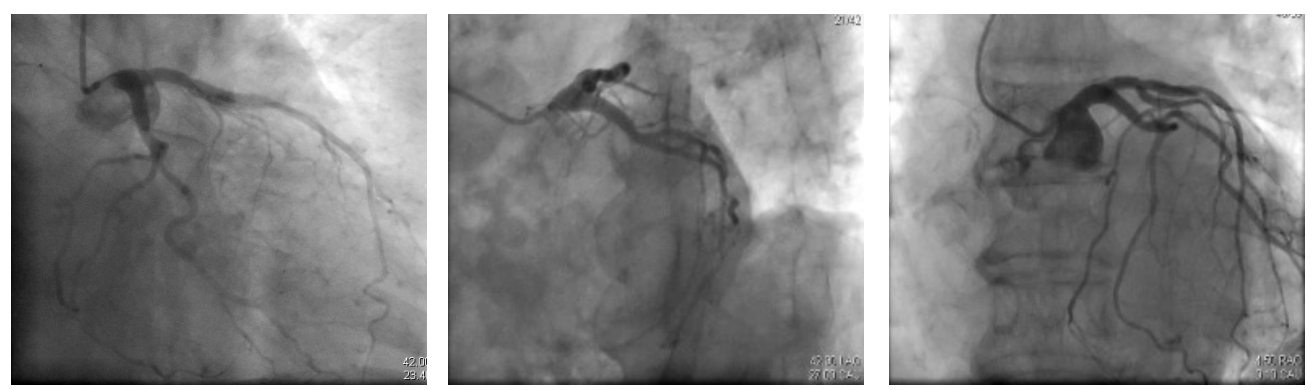

Figure 3. Coronary angiography at 6-mos follow-up from Case-1, demonstrating normal aortic bulb with complete stents patency and TIMI 3 flow of the LAD and LCX (Panel A/B and C). 


\section{Case report 2}

A 58-year-old non-smoker male with family history of coronary artery disease was referred because of progressive angina during moderate exercise and a positive stress test with $2 \mathrm{~mm}$ ST depression from lead V1 to V5. Coronary angiography performed through right radial access demonstrated a significant mid RCA stenosis (Figure 4 - Panel A) associated with severe left coronary system calcification, a critical stenosis of mid LCX (Figure 4 - Panel B), and a severe loop of the proximal LAD followed by critical stenosis of mid-distal LAD (Figure 4 - Panel C). The LM was catheterized with a $3.5 \mathrm{EBU} 6 \mathrm{Fr}$ guiding catheter, and a Balance Middleweight guidewire (Abbott Diagnostics, Lake Forest, Illinois) was placed in the distal LAD. After pre-dilatation with an Euphora $2.5 \times 20 \mathrm{~mm}$ PTCA balloon at 12 atm (Medtronic, Santa Rosa, CA, USA) we tried to implant a DES Onyx $3 \times 23 \mathrm{~mm}$ which was not able to cross the lesion. Thus, a $3 \times 12$ mm lithotripsy balloon (Shockwave Medical Inc) was placed across the lesion with application of 80 pulses at 4,6 and 8 atm (Figure $4-$ Panel D insert). At control angiography an antegrade and retrograde dissection of the entire LAD segment was evident (Figure 4 - Panel D) with involvement of LM (Dunning classification type II). The patient experienced acute chest pain and progressive hypotension requiring vasoactive drug support, emergency intubation and intra-aortic balloon pump (IABP) placement (Figure 4 - Panel E). Subsequently, also LCX occluded due to extension of the dissection flap to LCX ostium. This event was followed by ventricular fibrillation storm and development of severe cardiogenic shock. External heart massage followed by several DC shock were immediately deployed to the patient. Then, an Impella 2.5 heart pump device (Abiomed Inc., Danvers, MA, USA) was placed in the left ventricle for percutaneous mechanical circulatory support (Figure 4 - Panel F). However, cardiogenic shock persisted, and several ventricular fibrillation episodes continued to occur. Thus, a veno-arterial extracorporeal membrane oxygenation (VA-ECMO) was placed with immediate improvement of patient's hemodynamic and stabilization of the ventricular electric storm. A transthoracic echocardiography was then performed to establish the integrity of the aortic valve. The time interval for complete hemodynamic stabilization was almost 2.5 hours. At this point, the EBU catheter, lost during the reanimation manouvers, was re-advanced into the LM ostium and advancement of a Fielder XT-A wire (Asahi Intecc Co., Ltd. Aichi, Japan) through the true-false lumen was obtained with reestablishment of a TIMI-1 distal flow on LAD. The LM ostium and the entire LAD was then dilated with an Euphora balloon $3.5 \times 20 \mathrm{~mm}$ at $12 \mathrm{~atm}$ as in a "knuckle technique" fashion, restoring a distal TIMI 2 coronary flow (Figure 4 - Panel G-H). The LCX was re-opened with IVUS-guided mini subintimal and re-entry ("mini-STAR") technique and final implantation of a Resolute Onyx DES 3x12 mm (Medtronic, Minneapolis USA) at the origin of LCX. Procedure was then completed with positioning of a Resolute Onyx DES $3.0 \times 28 \mathrm{~mm}$ in the proximal part of the LAD. It was impossible to advance a second DES in the mid part of the LAD despite utilization of 6 Fr GuideLiner ${ }^{\infty}$ catheter (Vascular Solutions Inc., Minneapolis, Minnesota, United States) due to the marked tortuosity of the vessel. At the end of the procedure LM presented a small no-flow limiting dissection which was left untreated since judged safe. TIMI 2-3 flow in the distal bed of the LAD was present. The patient recovered for 2 weeks in the ICU and the VAECMO removed after one-week. Ejection fraction was $51 \%$ at $45^{\text {th }}$ days and the patient were discharged at $62^{\text {nd }}$ days. A coronary angiography performed 5 months for recurrence of chest pain with ECG ischemic modifications in anterior leads demonstrated complete sealing of the LM with good patency of the LAD and LCX stents and residual significant stenosis of mid LAD, mid LCX and an FFR of 0.69 on mid RCA. In addition, a double-rail no-limiting-flow dissection in the mid and distal segment of the LAD was still present (Figure 5). The heart team decided to increase medical therapy and to wait to allow better sealing of the LAD. However, the patient, after 8 months, was submitted to double coronary bypass (LIMA-IVA, RIVA-RCA) for recurrence of several episodes of angina. He is asymptomatic since. The $\mathrm{EF}$ is $51 \%$ by echo examination.

\section{Discussion and conclusion}

Iatrogenic coronary spiral dissection is rare and has been estimated to occur in approximately 0.02 to $0.08 \%$ of diagnostic catheterization and in 0.07 to $0.6 \%$ of PCIs [3-7]. Once coronary dissection occurs, it
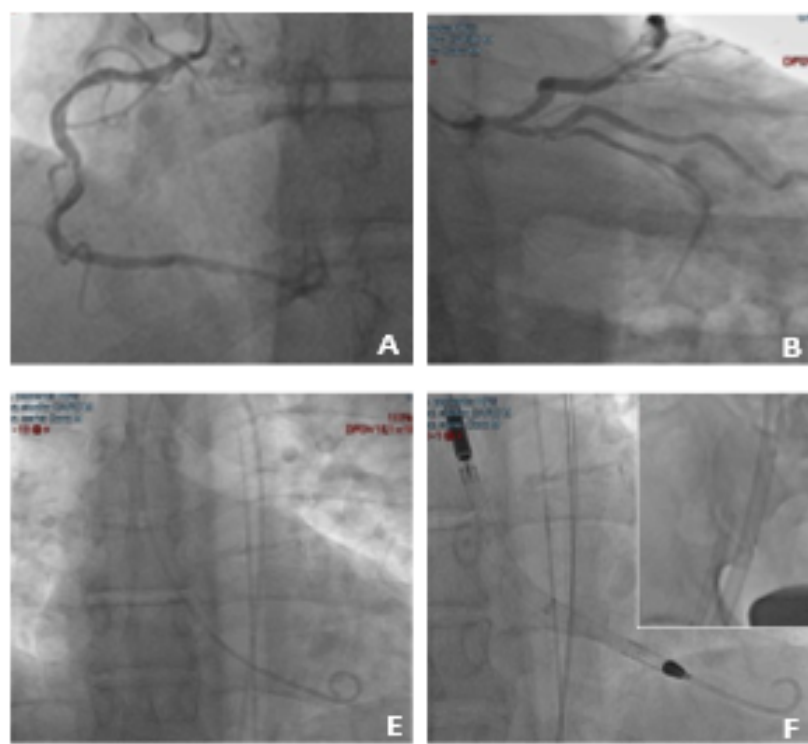
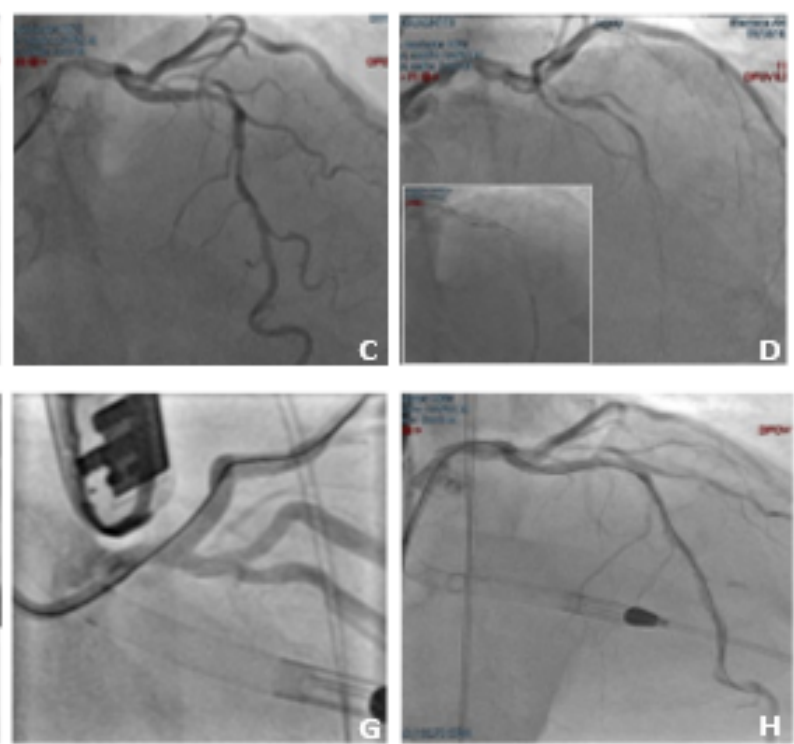

Figure 4. Case-2: coronary angiography demonstrating RCA with significative mid stenosis (Panel A), LM calcification with severe stenosis of the mid LCX (Panel B) and long, significant calcific stenosis of LAD (Panel C). Lithotripsy balloon across the lesion (insert of D), antegrade and retrograde dissection of the entire LAD segment with involvement of LM (Dunning classification type II) (Panel D). IABP insertion (Panel E). Impella 2.5 heart pump device followed by ECMO (box insertion) for mechanical circulatory support (Panel F). Re-wiring of true lumen of LAD after cannulation with EBU catheter and balloon dilation of the entire vessel, restoring a TIMI 2 coronary flow (Panel G-H). 

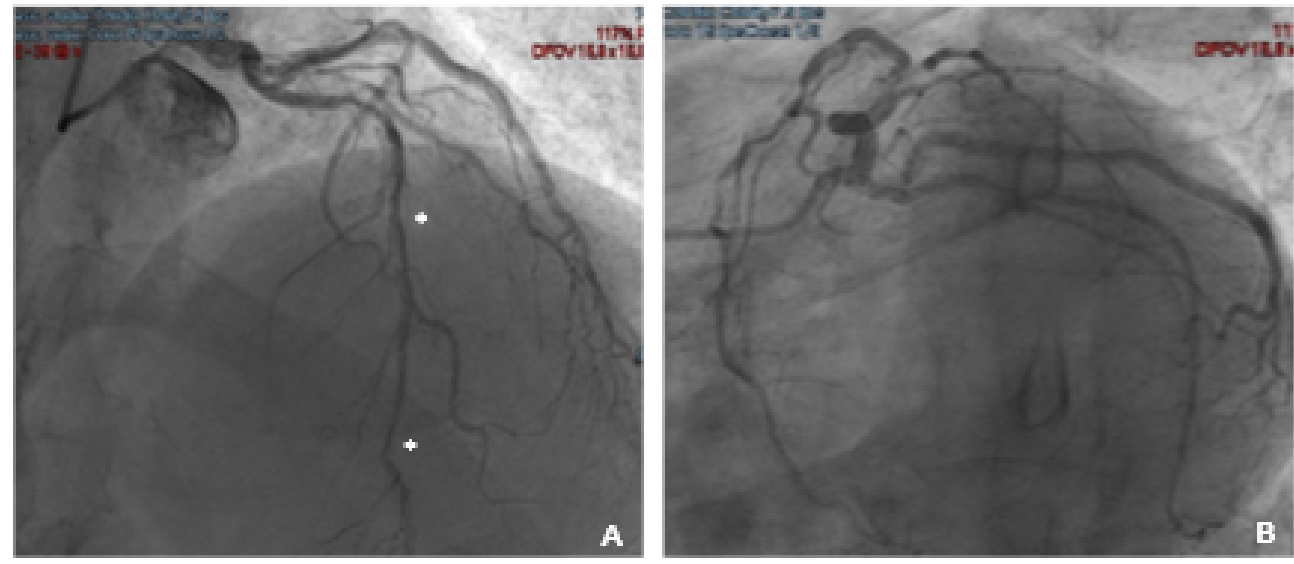

Figure 5. Case-2: five mos follow-up coronary angiography showed complete sealing of the LM with good patency of the LAD and LCX stents with a double-rail dissection not-limitingflow in the mid and distal segment of the LAD suggesting not complete healing of the dissection plane.

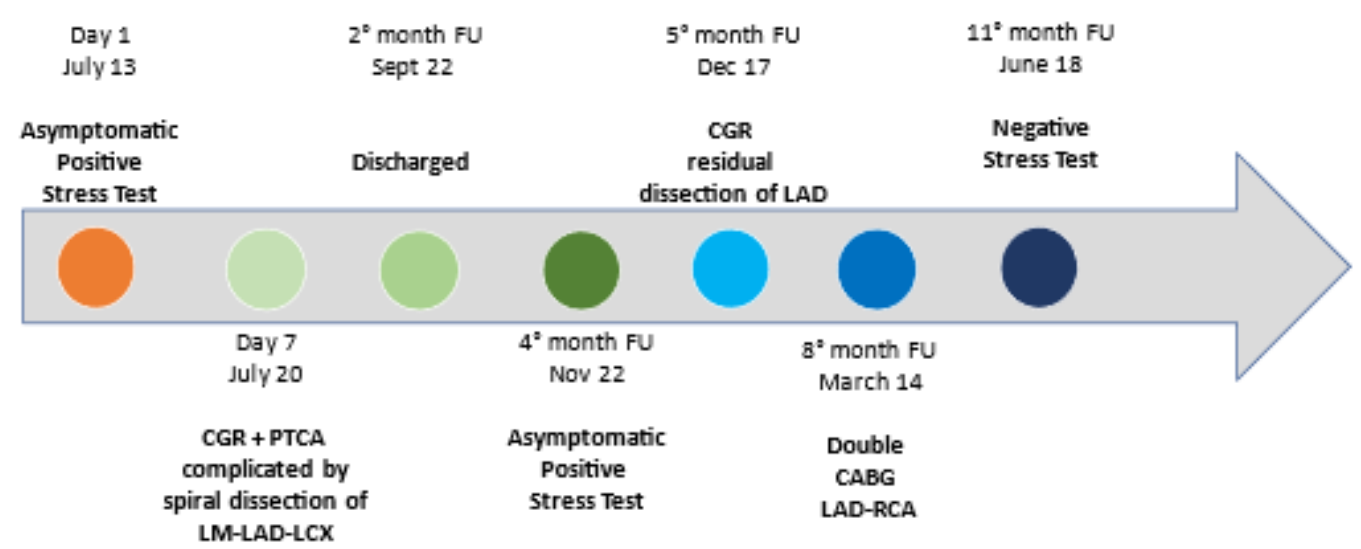

CASE 2

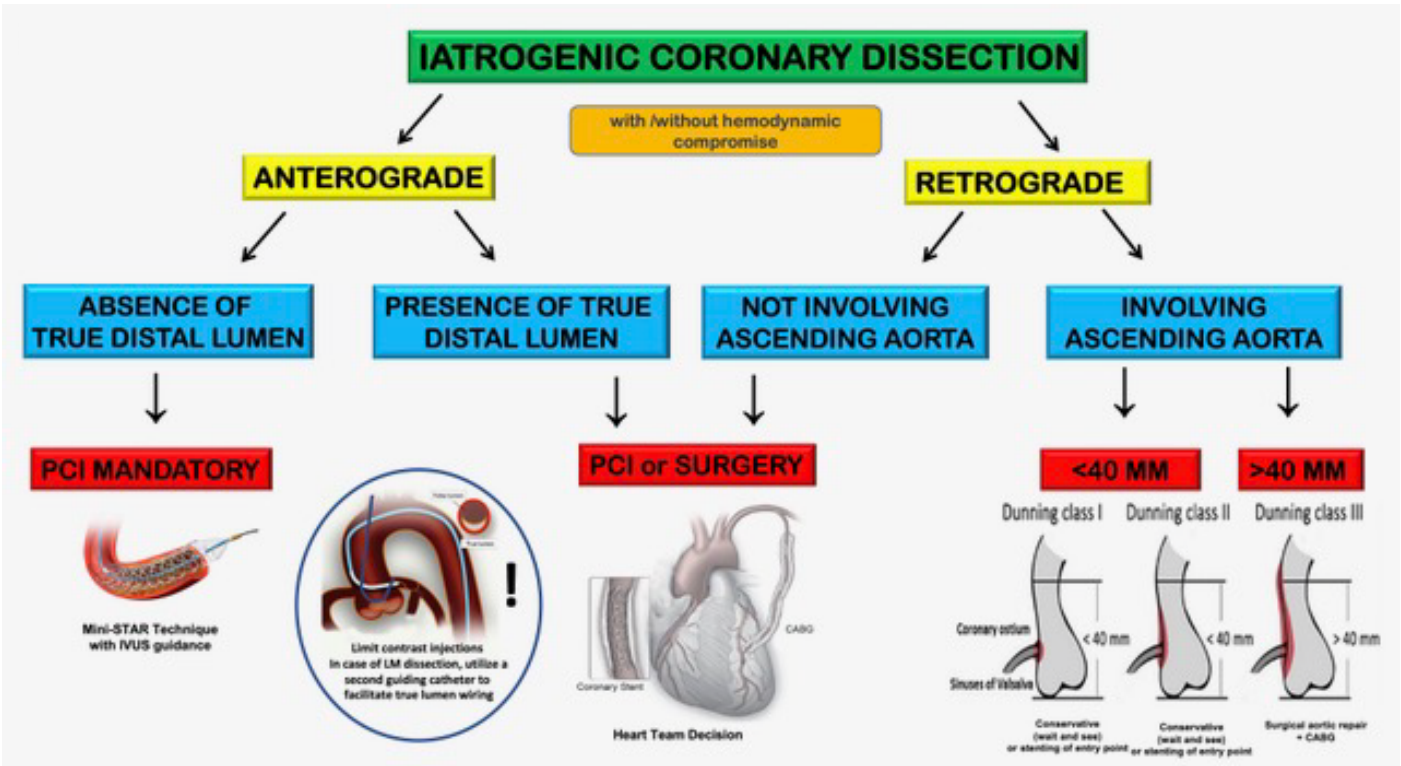

Figure 6. Management strategy algorithm of ICSD. If an antegrade dissection with absence of true distal lumen is present, it is mandatory for the team to keep trying to re-open the vessel by PCI since surgery may not succeed in finding true distal lumen. On the contrary, if true distal lumen is present, the decision strategy between PCI and CABG can be taken depending on dissection extension and involvement of side branches. In case of retrograde dissection involving aorta, PCI should be done if dissection involves either only the ostium of LM or $<40$ mm of the aorta. Conversely, surgery should be done for dissection involving $>40 \mathrm{~mm}$ of the ascending aorta. 
can rapidly propagate in an anterograde direction into the distal part of the coronary artery and, sometimes, in the opposite direction into the aorta [3]. In these latter cases, if the dissection is limited to a few centimetres beyond the aortic valve, it can be treated by sealing the entry point with a coronary stent [8]. Dunning et al. proposed a classification of iatrogenic coronary spiral dissection into 3 grades: type 1, limited to the sinuses of Valsalva; type 2, dissection of the ascending aorta beyond the sinuses but $<4 \mathrm{~cm}$; and type $3, \geq 4 \mathrm{~cm} 3$. A simplified and practical classification has been also proposed, based on the extension of the dissection flap: a localized, without extension into the LAD or LCX is defined as type I; extension of from the LM into the LAD or LCX is type II; and extension into the aortic root is classified as type III [9]. While type I dissections are associated with excellent outcomes, type III may have almost $100 \%$ in-hospital mortality [9]. In this setting, some authors previously reported that patients who experienced nonobstructive iatrogenic coronary spiral dissection at final angiography, had a worse prognosis compared to those without iatrogenic coronary spiral dissection (1-month composite of death, myocardial infarction, or target vessel revascularization: $12.2 \%$ vs $5.2 \%, \mathrm{p}=0.043$ ) [10].

The appropriate therapeutic strategy for iatrogenic dissections remains variable being generally based on the type of dissection and the hemodynamic stability of the patient. However, it is always useful to develop a mental algorithm for management strategy. First, since contrast media will preferentially circulate in the false lumen, the operator should immediately minimize injections due to the fact that further injections may promote expansion of the dissection. Thus, if contrast checks are needed, reduction of injection pressure $(<600$ PSI), injection flow $(<3 \mathrm{ml} / \mathrm{s})$ and contrast volume $(<5 \mathrm{ml})$, it is recommendable. Second, it is essential to be prepared for patient's hemodynamic destabilization. In the case examples we showed herein, Case-1 was characterized by hemodynamic stability which gave to the operating team the time to protect large branches originating from the LAD, seal the LAD-LM-aortic root dissection and finally re-open the LCX. In Case-2, abrupt hemodynamic compromise associated to VF storm made impossible to immediately re-open the left coronary three. In this situation, hemodynamic stabilization should be first accomplished and only after that, injury of the coronary artery sealed. In both cases it's suggested to perform angioplasty with an expert operator, as we did it in our experiences. Third, in order to re-open the vessels involved in the dissection, it is recommended to utilize intravascular ultrasound (IVUS) since it could help to confirm the correct wire position in the true lumen and to assess the extension of intramural haematomas [11]. However, once the dissection has occurred, the clinical picture may vary depending on the remaining antegrade coronary flow, and it can range from an asymptomatic patient, as in Case-1, to a patient in refractory cardiogenic shock, as in Case-2 [12,13]. Some authors have proposed that in all cases where there is dissection of the coronary sinus, the ostium should be sealed immediately by stenting [14]. Others have proposed that surgical intervention should be considered if the dissection extends to $4 \mathrm{~cm}$ or beyond into the aorta $[3,15]$. In case of LM dissection, it is also possible to maintain the guiding catheter into the false lumen and utilize a second guiding catheter to facilitate true lumen wiring [16]. A management strategy to treat iatrogenic coronary spiral dissection is proposed (Figure 6). It is important to remember that in case of anterograde dissection extending to distal vessel segments, it is difficult for the heart surgeon to choose the correct landing zone for the distal anastomosis and is therefore recommendable a "keep-calm and carry on" strategy in order to percutaneously re-open the vessel (usually with a "mini-STAR" technique). On the contrary, in the presence of true distal lumen, the heart team may decide to perform either PCI or CABG.

\section{Learning points}

- It is recommended the reduction of pressure, flow, volume and number of injections during coronary dissection.

- The interventional cardiologist should know how to manage the case in case of retrograde coronary dissection involving aorta based on Dunning classification, and different techniques such as miniSTAR and knuckle, in order to re-open the vessels in case of spiral antegrade coronary iatrogenic dissection.

- Intravascular ultrasound (IVUS) may help the operator to confirm the correct wire position in the true lumen in case of coronary dissection.

- Shock-wave balloon lithotripsy dilation may cause dissection of the vessel (as in case 2). The operator should be ready to switch strategy in order to solve this complication.

- In case of iatrogenic coronary dissection, a "keep calm and carry on" strategy should be adopted by the interventional cardiologist team.

\section{References}

1. Awadalla H, Sabet S, El Sebaie A, Rosales O, Smalling R (2005) Catheter-induced left main dissection incidence, predisposition and therapeutic strategies experience from two sides of the hemisphere. J Invasive Cardiol 17: 233-236. [Crossref]

2. Devlin G, Lazzam L, Schwartz L (1997) Mortality related to diagnostic cardiac catheterization. The importance of left main coronary disease and catheter induced trauma. Int J Card Imaging 13: 379-384. [Crossref]

3. Dunning DW, Kahn JK, Hawkins ET, O'Neill WW (2000) Iatrogenic coronary artery dissections extending into and involving the aortic root. Catheter Cardiovasc Interv 5: 387-93. [Crossref]

4. Galassi AR, Tomasello SD, Costanzo L, Campisano MB, Barrano G, et al. (2012) Mini-star as bail-out strategy for percutaneous coronary intervention of chronic total occlusion. Catheter Cardiovasc Interv 79 :30-40. [Crossref]

5. Carter AJ, Brinker JA (1994) Dissection of the ascending aorta associated with coronary angiography. Am J Cardiol 73: 922-923. [Crossref]

6. Li JC, Guan XL, Gong M, Zhang HJ (2018) Iatrogenic aortic dissection during percutaneous coronary intervention: A case report and review of the literature. $J$ Int Med Res 46: 526-532. [Crossref]

7. Hiraide T, Sawano M, Shiraishi Y, Ueda I, Numasawa Y, et al. (2018) Impact of catheter-induced iatrogenic coronary artery dissection with or without postprocedural flow impairment: A report from a Japanese multicenter percutaneous coronary intervention registry. PLoS One 13: e0204333. [Crossref]

8. Al-Saif SM, Liu MW, Al-Mubarak N, Agrawal S, Dean LS (2000) Percutaneous treatment of catheter-induced dissection of the left main coronary artery and adjacent aortic wall: a case report. Catheter Cardiovasc Interv 49: 86-89.

9. Eshtehardi P, Adorjan P, Togni M, Tevaearai H, Vogel R, et al. (2010) Iatrogenic left main coronary artery dissection: incidence, classification, management, and long-term follow-up. Am Heart $J$ 159: 1147-53. [Crossref]

10. Biondi-Zoccai GGL, Agostoni P, Sangiorgi GM, Airoldi F, Cosgrave J, et al. (2006) Incidence, predictors, and outcomes of coronary dissections left untreated after drugeluting stent implantation. Eur Heart J 27: 540-546. [Crossref]

11. Sanidas E, Buysschaert I, van Langenhove G (2014) Iatrogenic left main coronary artery dissection and intramural hematoma caused by diagnostic transradial cardiac catheterization. Hellenic J Cardiol 55: 65-69. [Crossref]

12. Shah P, Bajaj S, Shamoon F (2016) Aortic dissection caused by percutaneous coronary intervention: 2 new case reports and detailed analysis of 86 previous cases. Tex Heart Inst J 43: 52-60. [Crossref]

13. El-Haress M, Daadaa H, Shahjouei S, El-Bitar F, Bahmad H (2017) Iatrogenic Acute Ascending Aortic Dissection with Intramural Hematoma during Coronary Artery Stenting: A Case Report. Front Surg 4: 2. [Crossref]

14. Carstensen S, Ward MR (2008) Iatrogenic aortocoronary dissection: the case for immediate aortoostial stenting. Heart Lung Circ 17: 325-329. [Crossref] 
Iorio R (2021) Case reports of antegrade and retrograde iatrogenic coronary spiral dissection complicating percutaneous coronary intervention: Keep calm and carry on!

15. Sohrabi B, Kazemi B, Aslanabadi N (2007) Percutaneous treatment of catheter-induced dissection of the right coronary artery and adjacent aortic wall. $J$ Invasive Cardiol 19 : E199-E202. [Crossref]
16. Prakash R, Starovoytov A, Heydari M, Mancini GB, Saw J (2016) Catheter-Induced Iatrogenic Coronary Artery Dissection in Patients with Spontaneous Coronary Artery Dissection. JACC Cardiovasc Interv 9: 1851-1853. [Crossref]

Copyright: (C2021 Iorio R. This is an open-access article distributed under the terms of the Creative Commons Attribution License, which permits unrestricted use, distribution, and reproduction in any medium, provided the original author and source are credited. 